SHORT COMMUNICATION

\title{
Differences in GATA3 EXPRESSiOn AMONG HistologicAl/ \\ MOLECULAR SUBTYPES AND GRADES IN INFILTRATING BREAST \\ CARCINOMA (IBC) ARE IMPORTANT IN THE DIAGNOSIS OF \\ METASTATIC BREAST CARCINOMA
}

Simona Stolnicu ${ }^{1}$, Csillip Tunde ${ }^{1}$, Ana Cadar ${ }^{1}$, Monica Boros $^{2}$

${ }^{1}$ Department of Pathology, University of Medicine, Pharmacy, Sciences and Technology (UMFTS) of Târgu Mureş, Romania

${ }^{2}$ Department of Pathology, Faculty of Medicine and Pharmacy, University of Oradea, Romania

\begin{abstract}
GATA-binding protein 3 (GATA3) is a sensitive and relatively specific marker in invasive breast carcinomas (IBC). The aim of the present study was to determine GATA3 expression among different histological and surrogate molecular breast carcinoma subtypes.

Immunohistochemical staining of ER, PR, Ki67, HER2 and GATA3 was performed in a cohort of 84 consecutive cases of primary IBCs. The association of GATA3 expression with different histological subtypes, grades and surrogate intrinsic molecular subtypes was assessed.

The overall positivity of GATA3 across various histological subtypes was $71.43 \%$ with no correlation with histological type $(\mathrm{p}=0.849)$. Our study also confirmed that GATA3 exhibits a relatively high sensitivity for IBC (78.57\%). GATA3 expression was positively correlated with low histological grades (G1/G2) vs. G3: $\mathrm{p}=0.001)$ with most of G3 $(57.89 \%)$ cases being negative and with luminal A $(72.22 \%)$ and $\mathrm{B}(84.62 \%)$ subtypes $(\mathrm{p}=0.00001)$ while most of the triple negative $(87.5 \%)$ and HER2-overexpressed $(66.67 \%)$ being negative.

Caution must be payed however when dealing with an IBC metastasis of HER2-overexpressed or triple negative molecular subtypes or G3, since in these categories according to the present study, GATA3 is more frequently negative that previously reported and may be useless for diagnosis of tumor origin.
\end{abstract}

Key words: infiltrating breast carcinoma, GATA3, histologic, molecular subtypes.

GATA-binding protein 3 (GATA3) is a well-studied transcription factor found to be essential in the development of luminal breast epithelium. Moreover, GATA3 was proposed as a sensitive and relatively specific marker in primary IBC as well as for primary genital extramammary paget disease $[1]$. Its diagnostic and prognostic utility in primary IBC has been confirmed although by limited studies revealing that high expression of GATA3 is associated with posi- tive estrogen (ER) and progesterone (PR) receptors tumors, smaller tumor size and lower nuclear grade, and as a consequence with a better survival while loss of GATA3 expression was found to be associated with unfavorable outcome [2]. This finding is not universally accepted as published data on GATA3 as a prognostic markers are conflicting [3]. We read with interest the paper by Shaoxian et al. on characterization of GATA 3 expression in IBC [4]. In that 
particular study, on a larger cohort of patients with primary IBC, the authors demonstrated that GATA3 exhibits a relatively high sensitivity for IBCs, being more sensitive than GCDFP15 and mammaglobin in luminal-like and HER2 overexpression subtypes, and being more likely associated with IBC of luminal subtype and low histologic grade. Similar results were found by $\mathrm{Ni}$ et al. on histologic specimens as well as by Hafez et al. and El Hag et al. on cytological cell block materials $[5,6,7]$. Of interest, the negative rate for GATA3 was recorded as being of $28 \%$ in grade 3 tumors by the recent study of Lu et al. with more GATA3 negative tumors occuring in grade 3 of no special type, metaplastic and apocrine IBCs [3]. Consequently, in the routine practice, especially when dealing with a metastatic IBC, the role of GATA3 may be limited. We aimed to further investigate the role of GATA3 expression among different histological subtypes, surrogate molecular IBC subtypes and histological grades in a prospective study. Immunohistochemical staining of ER, PR, ki67, HER2 and GATA3 was performed in a cohort of 84 consecutive cases of primary IBCs. The association of GATA3 expression with different histological subtypes, grades and surrogate intrinsic molecular subtypes was assessed $[8,9]$.

The age ranged from 35 to 83 years with median age of 61.9 years (no statistically significant differences between with median age in GATA3 positive and negative cases and between pre or postmenopausal IBC patients) $(\mathrm{p}=0.832)$. The overall positivity of GATA3 across various histological subtypes was $71.43 \%$ with no correlation with histological type $(\mathrm{p}=0.849)$. GATA3 expression was positively correlated with low histological grades (G1/G2 vs. G3: $p=0.001)$ with most of G3 $(57.89 \%)$ cases being negative and with luminal A $(72.22 \%)$ and B (84.62\%) subtypes $(p=0.00001)$ while most of

Table I. Correlation between GATA3 expression and age, histological type and grade and molecular profile

\begin{tabular}{|c|c|c|c|c|c|c|c|}
\hline \multirow[t]{2}{*}{ PARAmeter } & \multicolumn{5}{|c|}{ GATA } & \multirow[b]{2}{*}{$\%$} & \multirow[b]{2}{*}{ OR, CI, P } \\
\hline & $\mathbf{N}$ & $\%$ & POSITIVE & $\%$ & NEGATIVE & & \\
\hline Total & 84 & & 60 & 71.4 & 24 & 28.57 & \\
\hline Age median & 61.9 & & \multicolumn{2}{|c|}{63.08} & \multicolumn{2}{|c|}{58.95} & \\
\hline Range & $35-83$ & & \multicolumn{2}{|c|}{$39-83$} & \multicolumn{2}{|c|}{$35-76$} & \\
\hline Age $<50$ years & 13 & 15.48 & 9 & 69.23 & 4 & 30.77 & Age $<50$ years vs. age $\geq$ \\
\hline \multirow[t]{2}{*}{ Age $\geq 50$ years } & \multirow[t]{2}{*}{71} & \multirow[t]{2}{*}{84.52} & \multirow[t]{2}{*}{51} & \multirow[t]{2}{*}{71.83} & \multirow[t]{2}{*}{20} & \multirow[t]{2}{*}{28.17} & 50 years \\
\hline & & & & & & & $\begin{array}{c}\mathrm{OR}=0.832,95 \% \mathrm{CI}: \\
0.248-3.070 \\
\mathrm{p}=0.832\end{array}$ \\
\hline \multicolumn{8}{|l|}{ Histologic type } \\
\hline NST & 71 & 84.52 & 51 & 71.83 & 20 & 28.17 & \multirow{7}{*}{$\begin{array}{c}\text { Histologic type } \\
\text { NST vs. others } \\
\text { OR }=1.133 \\
95 \% \text { CI: } 0.313-4.102 \\
\mathrm{p}=0.849\end{array}$} \\
\hline Lobular & 8 & 9.52 & 6 & 70.00 & 2 & 25.00 & \\
\hline Apocrine & 1 & 1.19 & 0 & 75.00 & 1 & 100.00 & \\
\hline Micropapillary & 1 & 1.19 & 1 & 0.00 & 0 & 0.00 & \\
\hline Papillary & 1 & 1.19 & 1 & 100.00 & 0 & 0.00 & \\
\hline Mucinous & 1 & 1.19 & 1 & 100.00 & 0 & 0.00 & \\
\hline Mixed & 1 & 1.19 & 0 & 100.00 & 1 & 100.00 & \\
\hline \multicolumn{7}{|l|}{ Histologic grade } & \multirow{5}{*}{$\begin{array}{l}\text { Histological grade } 1.2 \text { vs. } 3 \\
\qquad \begin{array}{c}\text { OR }=5.747 \\
\begin{array}{c}95 \% \mathrm{CI}: 1.869-17.543 \\
\mathrm{p}=0.001\end{array}\end{array}\end{array}$} \\
\hline G1 & 13 & 15.48 & 12 & 92.31 & 1 & 7.69 & \\
\hline G2 & 44 & 52.38 & 34 & 77.27 & 10 & 22.73 & \\
\hline G3 & 19 & 22.62 & 8 & 42.11 & 11 & 57.89 & \\
\hline N/A & 8 & 9.52 & 6 & 75.00 & 2 & 25.00 & \\
\hline Luminal A & 18 & 21.43 & 13 & 72.22 & 5 & 27.78 & \multirow{2}{*}{$\begin{array}{l}\text { Molecular Type Luminal } \\
\text { A,B vs. HER2+,TRN }\end{array}$} \\
\hline Luminal B & 52 & 61.90 & 44 & 84.62 & 8 & 15.38 & \\
\hline $\begin{array}{l}\text { HER2 } \\
\text { overexpressed }\end{array}$ & 6 & 7.14 & 2 & 33.33 & 4 & 66.67 & $\begin{array}{c}\text { OR }=17.544 \\
95 \% \text { CI: } 4.202-71.429\end{array}$ \\
\hline Triple negative & 8 & 9.52 & 1 & 12.50 & 7 & 87.50 & $\mathrm{p}=0.00001$ \\
\hline
\end{tabular}



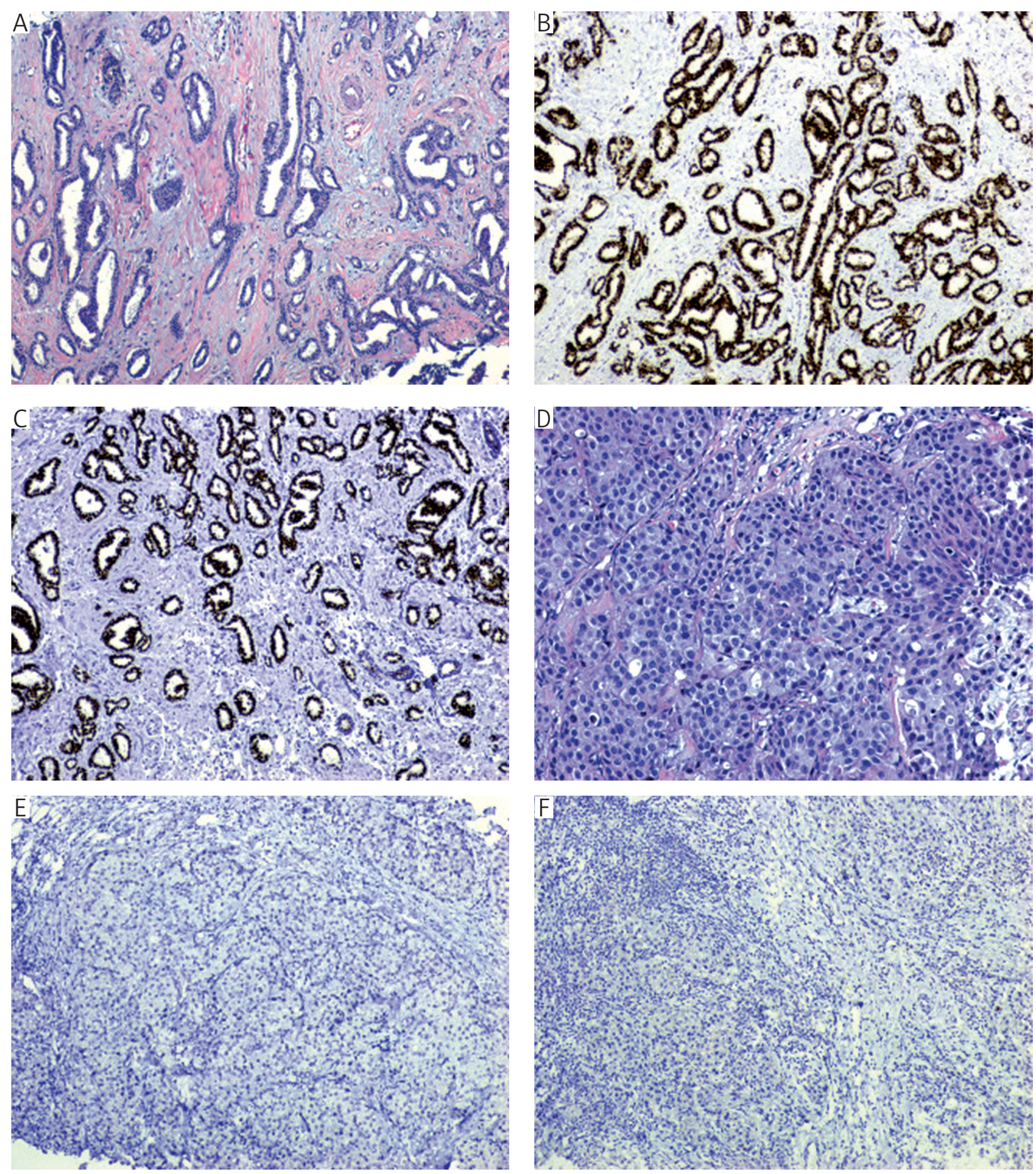

Fig. 1. Infiltrating breast carcinoma of no special type, histologic grade 1 (haematoxylin-eosin) (A), positive for ER (B) and GATA3 (C); Infiltrating breast carcinoma of no special type, histologic grade 3 (haematoxylin-eosin) (D), negative for ER (E) and GATA3 (F)

the triple negative $(87.5 \%)$ and HER2-overexpressed $(66.67 \%)$ being negative for GATA3 (Table I; Fig. 1). Our study confirmed that GATA3 exhibits a relatively high sensitivity for IBC (78.57\%). GATA3 expression is associated with IBC most frequently encountered histological subtypes with luminal A/B molecular subtype and low G1/G2 histological grade. Caution must be payed however when dealing with an IBC metastasis of HER2-overexpressed or triple negative molecular subtypes or G3, since in these categories according to the present study, GATA3 is more frequently negative that previously reported and may be useless for diagnosis of tumor origin.

The authors declare no conflict of interest. 


\section{References}

1. Zhao M, Zhou L, Sun L, et al. GATA 3 is a sensitive marker for primary genital extramammary paget disease: an immunohistochemical study of 72 cases with comparison to gross cystic disease fluid protein 15. Diagnostic Pathol 2017; 12: 51.

2. Guo Y, Yu P, Liu Z, et al. Prognostic and clinicopthologic value of GATA binding protein 3 in bresat carcer: a systematic review and meta-analysis. PLoS One 2017; 12: e0174843.

3. Lu S, Yakirevich E, Wang LJ, et al. Cytokeratin 7-negative and GATA binding protein 3-negative breast cancers: clinicopathological features and prognostic significance. BMC Cancer 2019; 19: 1085 .

4. Shaoxian T, Baohua Y, Xiaoli X, et al. Characterisantion of GATA 3 expression in invasive breast cancer: differences in histological subtypes and immunohistochemically defined molecular subtypes. J Clin Pathol 2017; 70: 926-934.

5. Ni YB, Tsang JYS, Shao MM, et al. GATA-3 is superior to GCDFP-15 and mammaglobin to identify primary and metastatic breast cancer. Breast Cancer Res Treat 2018; 169: 25-32.

6. Hafez NH, Shaaban HM. Can GATA3 immunohistochemistry be utilized as a reliable diagnostic marker for metastatic breast carcinoma in cytological material? A comparative study with Mammaglobin and GCDFP-15 expression. Turk Patholoji Derg 2018; 34: 143-149.

7. El Hag MI, Hag AM, Ha JP, et al. Comparison of GATA-3, mammaglobin, GCDFP-15 expression in breast carcinoma in serous effusions: A cell-block micro-array study. Pleura Peritoneum 2017; 2: 143-148.

8. Lakhani S, Ellis IO, Schnitt SJ, et al. WHO Classification of Tumours of the Breast. IARC press, Lyon 2012; 10-71.

9. Amin MB, Edge SB, Greene FL, et al. (eds.). AJCC cancer staging manual. 8th edition 2017.

\section{Address for correspondence}

\section{Simona Stolnicu}

Department of Pathology

University of Medicine, Pharmacy,

Sciences and Technology (UMFTS) of Târgu Mureş

Gheorghe Marinescu Street Nr 38

540139 Targu Mures, Romania

e-mail: stolnicu@gmx.net 